\title{
PERTURBED ASYMPTOTICALLY STABLE SETS
}

\author{
ROGER C. MCCANN
}

\begin{abstract}
Perturbations of a dynamical system are defined and the behavior of compact asymptotically stable sets under these perturbations is determined. The occurrence of critical points in a perturbed planar dynamical system is also investigated.
\end{abstract}

In [1] it is shown that if $C$ is an asymptotically stable cycle of a planar dynamical system $\pi$ and if $\pi_{i}$ is a net of planar dynamical systems which converges to $\pi$, then there are limit cycles $C_{i}$ of $\pi_{i}$ such that $C_{i} \rightarrow C$. This paper presents a similar result in a more general setting for perturbed asymptotically stable sets. If $\pi_{i}$ is a net of dynamical systems which converges to a dynamical system $\pi$ and if $M$ is a compact asymptotically stable set of $\pi$, then eventually there are asymptotically stable sets $M_{i}$ of $\pi_{i}$ arbitrarily close to $M$. Moreover, if $M$ is invariant with respect to all $\pi_{i}$, then $M_{i} \rightarrow M$.

$R, R^{+}$, and $R^{-}$will denote the reals, nonnegative reals, and nonpositive reals respectively.

A dynamical system $\pi$ on a topological space $X$ is a mapping of $X \times R$ onto $X$ which satisfies the following three conditions (where $x \pi t=$ $\pi(x, t))$ :

(i) $\pi$ is continuous in the product topology.

(ii) $x \pi 0=x$ for each $x \in X$.

(iii) $(x \pi t) \pi s=x \pi(t+s)$ for each $x \in X$ and $s, t \in R$.

If $A \subset X$ and $B \subset R$, then $A \pi B$ will denote the set $\{x \pi t: x \in A, t \in B\}$. $L^{+}(x)$ and $L^{-}(x)$ will denote the positive limit set of $x$ and the negative limit set of $x$ respectively. A subset $M$ of $X$ is called an (negative) attractor iff there is a neighborhood $U$ of $M$ such that $\left(L^{-}(x)\right) L^{+}(x) \subset M$ for every $x \in U$. If $M$ is an (negative) attractor, then $\left(A^{-}(M)\right) A^{+}(M)$ will denote the largest such neighborhood.

A subset $S$ of $X$ is called a section with respect to $\pi$ iff $(S \pi t) \cap S=\varnothing$ for all $t \neq 0$.

In a topological space $X$ it is possible to define limits of nets of subsets

Received by the editors April 12, 1971 and, in revised form, January 7, 1972.

AMS 1970 subject classifications. Primary 34C35, 34D10; Secondary 34C05.

Key words and phrases. Dynamical systems, perturbation asymptotic stability, stable critical point.

c. American Mathematical Society 1972 
$X_{i} \subset X$ as follows: let lim inf $X_{i}$ consist of all limits of nets of points $x_{i} \in$ $X_{i}$; let lim sup $X_{i}$ consist of all limits of subnets of points $x_{i} \in X_{i}$. Obviously $\lim \inf X_{i} \subset \lim \sup X_{i}$. If equality holds, the net $X_{i}$ is said to converge and we write

$$
\lim X_{i}=\lim \inf X_{i}=\lim \sup X_{i}
$$

Let $\pi$ be a dynamical system on $X$ and $\pi_{i}$ be a net of dynamical systems on $X$ such that $\pi_{i} \rightarrow \pi$ in the following sense: if $x_{j}$ and $t_{j}$ are nets converging to $x$ and $t$ respectively, then $x_{j} \pi_{i} t_{j} \rightarrow x \pi t$ [2, VI, 3.1-3.11]. If $X$ is locally compact, then the convergence of $\pi_{i}$ to $\pi$ as defined above is equivalent to the convergence of $\pi_{i}$ to $\pi$ in the compact open topology [2, VI, 3.3]. A section with respect to $\pi$ may not be a section with respect to any of the $\pi_{i}$ [2, VI, 3.10.1].

Convention. Any set subscripted by an $i$ is to be considered relative to $\pi_{i}$; e.g., $L_{i}^{+}(x)$ is the positive limit set of $x$ with respect to $\pi_{i}$.

The purpose of this paper is to prove the following theorem.

THEOREM 1. Let $X$ be a locally compact metric space on which there is defined a dynamical system $\pi$ and a net $\pi_{i}$ of dynamical systems such that $\pi_{i} \rightarrow \pi$. If $M$ is a compact asymptotically stable set of $\pi$, then there are asymptotically stable sets $M_{i}$ of $\pi_{i}$ such that $\lim \sup M_{i} \subset M$. Moreover, if $M$ is invariant with respect to each $\pi_{i}$, then $\lim M_{i}=M$.

Since $M$ is asymptotically stable there is a continuous Liapunov function $v: A^{+}(M) \rightarrow R^{+}$for $M$ such that (i) $v(x \pi t)<v(x)$ whenever $x \notin M$ and $t>0$ and (ii) $v(x)=0$ whenever $x \in M[3$, Theorem 10]. $\mathscr{F}$ will denote the family $\left\{v^{-1}([0, r]): r>0\right\}$, which is a fundamental system of neighborhoods of $M$. It is easily verified that $v^{-1}(r), r>0$, is a section with respect to $\pi$.

The proof of the theorem depends on the following two lemmas.

LEMMA 2. Let $U, V \in \mathscr{F}$ be such that both are compact and $V \subset$ int $U$. Then eventually $(\mathrm{cl}(X-U)) \pi_{i} R^{-} \subset X-V$.

Proof. Set $A=X-U$ and $B=X-V$. Evidently both are open and $\bar{A} \subset B$. Let $\alpha<0$. By the construction of $U$, we have $\partial A \pi \alpha \subset A$ and $\bar{A} \pi_{i}[2 \alpha, 0] \subset \bar{A} \subset B$. Since $\pi_{i} \rightarrow \pi$, eventually, say $i>i_{0}, \partial A \pi_{i} \alpha \subset A$ and $\bar{A} \pi[2 x, 0] \subset B$. We now show that $\bar{A} \pi_{i} R^{-} \subset B$ for $i>i_{0}$. Assume not. Then there is an $x \in \partial A$ and $t \in R^{-}$such that $x \pi_{i} t \in \partial B$. Set $s=$ $\inf \left\{\tau: x \pi_{i} \tau \in \partial A, t<s \leqq 0\right\}$. Then $t<s$ and $x \pi_{i} s \in \partial A$ since $\partial A$ is compact. Moreover, $x \pi_{i}(t, s) \cap \bar{A}=\varnothing$. Since $x \pi_{i} s \in \partial A$ and $x \pi_{i} t \in \partial B$, we have that $t-s<2 \alpha$ (recall $\bar{A} \pi_{i}[2 \alpha, 0] \subset B$ for $i>i_{0}$ ). But $\partial A \pi_{i} \alpha \subset$ int $A$. This contradicts $\varnothing=x \pi_{i}(t, s) \cap \bar{A}=\left(\left(x \pi_{i} s\right) \pi(t-s, 0)\right) \cap \bar{A}$. This contradiction implies $\bar{A} \pi_{i} R^{-\subset B}$ for $i>i_{0}$. 
Lemma 3. Let $U \in \mathscr{F}$ be compact. Then $\operatorname{cl}(X-U)$ is eventually a negative attractor with respect to $\pi_{i}$.

Proof. Let $V, W \in \mathscr{F}$ be compact and such that $V \subset$ int $U \subset U \subset$ int $W$ and set $A=X-W, B=X-U, C=X-V$. Each is open and $\bar{A} \subset B \subset \bar{B} \subset C$. Since $W$ and $V$ are in $\mathscr{F}$, for each $x \in \operatorname{cl}(C-A)$ there is a $t(x) \in R^{-}$such that $x \pi t(x) \subset A$. We will first show that there is a $T \in R^{-}$such that $x \pi[T, 0] \cap A \neq \varnothing$ for each $x \in \operatorname{cl}(C-A)$. Assume there is no such $T$. Then there are nets $x_{i}$ in $\operatorname{cl}(C-A)$ and $s\left(x_{i}\right)$ in $R^{-}$such that $s\left(x_{i}\right) \rightarrow-\infty$ and $x_{i} \pi\left[s\left(x_{i}\right), 0\right] \cap A=\varnothing$. Since $\operatorname{cl}(C-A)$ is compact, we may assume that $x_{i} \rightarrow x \in \operatorname{cl}(C-A)$. Then $x \pi t(x) \in A$ and, since $A$ is open and $\pi$ continuous, $x_{i} \pi t(x) \in A$ eventually. This contradiction implies the existence of a $T \in R^{-}$such that $x \pi[T, 0] \cap A \neq \varnothing$ for every $x \in \operatorname{cl}(C-A)$. Since $\operatorname{cl}(C-A)$ is compact and $\pi_{i} \rightarrow \pi$, eventually, say $i>i_{0}, x \pi_{i}[T, 0] \cap A \neq \varnothing$ for each $x \in \operatorname{cl}(C-A) . C$ is a neighborhood of $B . \bar{A} \pi_{i} R^{-} \subset B$ eventually (Lemma 2) so that $L_{i}^{-}(A) \subset B$. If $x \in \operatorname{cl}(C-A)$, then for $i>i_{0}, x \pi_{i}[T, 0] \cap A \neq \varnothing$ and (by Lemma 2) $L_{i}^{-}(\mathrm{cl}(C-A)) \subset B$ eventually. Thus eventually $L_{i}^{-}(C) \subset B$ and $B$ is eventually a negative weak attractor with respect to $\pi_{i}$.

Proof of Theorem 1. Let the notation be as in Lemma 3. $B$ is a negative attractor and $A_{i}^{-}(B)-B \subset U$. Therefore $\partial A_{i}^{-}(B) \subset U$ and $\partial A_{i}^{-}(B)$ is compact. Hence $X-A_{i}^{-}(B)$ is asymptotically stable with respect to $\pi_{i}[\mathbf{4}$, Theorem 3.10]. Thus we have shown that, for each $r, M(r)=X-v^{-1}([0, r])$ is eventually a negative attractor, $X-A_{i}^{-}(M(r))$ is asymptotically stable and $X-A_{i}^{-}(M(r)) \subset v^{-1}([0, r])$. Set $r_{i}=\inf \{r: M(r)$ is a negative attractor with respect to $\left.\pi_{i}\right\}$ and let $0 \leqq \varepsilon_{i} \leqq r_{i}$ be such that $M\left(r_{i}+\varepsilon_{l}\right)$ is a negative attractor with respect to $\pi_{i}$. Finally set $M_{i}=X-A_{i}^{-}\left(M\left(r_{i}+\varepsilon_{i}\right)\right) . M_{i} \subset$ $v^{-1}\left(\left[0, r_{i}+\varepsilon_{i}\right]\right)$ and is asymptotically stable. Lemma 3 implies $r_{i} \rightarrow 0$. Hence $M_{i} \subset v^{-1}\left(\left[0, r_{i}+\varepsilon_{i}\right]\right) \rightarrow v^{-1}(0)=M$, so that $\lim \sup M_{i} \subset M$. If $M$ is invariant with respect to each $\pi_{i}$, then $L_{i}^{+}(M) \subset M$ so that $M \subset X-$ $A_{i}^{-}\left(M\left(r_{i}+\varepsilon_{i}\right)\right)=M_{i}$. It easily follows that $M_{i} \rightarrow M$. This completes the proof.

REMARK. It should be noted that the converse of Theorem 1 is false. That is, if $M_{i}$ are compact asymptotically stable sets of $\pi_{i}$ and if $M_{i}$ converges to a compact set $M$, then it does not necessarily follow that $M$ is asymptotically stable with respect to $\pi$. Let $\pi$ be a planar dynamical system with the origin as a center-focus and $C_{n}(n=1,2, \cdots)$ a sequence of external limit cycles which converge to the origin. cl(int $C_{n}$ ) is asymptotically stable and $\lim \operatorname{cl}\left(\right.$ int $\left.C_{n}\right)$ is the origin. Finally for each positive integer $n$, set $\pi_{n}=\pi$. cl(int $C_{n}$ ) is asymptotically stable with respect to $\pi_{n}$, but the origin is not asymptotically stable with respect to $\pi$.

We now assume that $X$ is the plane $R^{2}$ and investigate the occurrence of critical points in the $\pi_{i}$. We will prove the following theorem. 
THEOREM 4. Let $x$ be a stable isolated critical point of a planar dynamical system $\pi$. Then there are critical points $x_{i}$ of $\pi_{i}$ such that $x_{i} \rightarrow x$.

The proof depends on the following three lemmas.

LEMMA 5. Each stable isolated critical point possesses arbitrarily small neighborhoods bounded by either a cycle or a section with respect to $\pi$ which is a simple closed curve.

Proof. The proof follows immediately from [2, VIII, 4.1] and [2, VIII, 4.3].

LeMMa 6. Let $x \in X$ possess a fundamental system $\mathscr{F}$ of neighborhoods whose boundaries are simple closed curves which are sections with respect to $\pi$. If $W$ is any neighborhood of $x$, then there is a neighborhood $V \subset W$ of $x$ such that eventually $L_{i}^{+}(V) \subset W$ or $L_{i}^{-}(V) \subset W$.

Proof. Let $U \in \mathscr{F}$. For $\varepsilon>0, \bar{U} \pi[\varepsilon,+\infty) \subset$ int $U$ or $\bar{U} \pi(-\infty,-\varepsilon] \subset$ $U$ [2, VII, 4.8]. Hence $\mathscr{F}$ contains a fundamental system $\mathscr{G}$ of compact neighborhoods of $x$ which consists entirely of positively invariant sets or of negatively invariant sets. For definiteness we will assume $\mathscr{G}$ consists of positively invariant sets. Let $V, U \in \mathscr{G}$ be such that $\bar{V} \subset$ int $U \subset \bar{U} \subset$ int $W$. In a manner similar to that used in the proof of Lemma 2 , it can be shown that eventually $V \pi_{i} R^{+} \subset U$. Thus $L_{i}^{+}(V) \subset \bar{U} \subset W$. If $\mathscr{G}$ consists of negatively invariant sets, the proof is analogous.

LEMMA 7. Let $x \in X$ possess a fundamental system $\mathscr{F}$ of neighborhoods whose boundaries are cycles of $\pi$. If $W$ is any simply connected neighborhood of $x$, then there are sets $V_{i} \subset W$ such that eventually either $L_{i}^{+}\left(V_{i}\right) \subset W$ or $L_{i}^{-}\left(V_{i}\right) \subset W$.

Proof. Let $U \in \mathscr{F}$ be such that $\bar{U} \subset$ int $W$. Since $\partial U$ contains no critical points with respect to $\pi$, eventually $\partial U$ contains no critical points with respect to $\pi_{i}[2, \mathrm{VI}, 3.7]$. Let $x_{0} \in \partial U, T$ be the fundamental period of $x_{0}$ with respect to $\pi$, and $S_{i}$ be transversals (local sections which are arcs) with respect to $\pi_{i}$ and which generate neighborhoods of $x$ ([2, VI, 2.12] and $[2, \mathrm{VII}, 1.6])$. Let $0<\varepsilon<\frac{1}{4} T$. Eventually $x_{0} \pi_{i}\left(0, \frac{1}{2} T\right] \cap S_{i}=\varnothing$, $x_{0} \pi_{i}\left[\frac{1}{2} T, \frac{3}{2} T\right] \cap S_{i} \pi_{i}[-\varepsilon, \varepsilon] \neq \varnothing, \quad$ and $\quad x \pi_{i}\left[0, \frac{3}{2} T\right] \subset$ int $W$. Set $t_{i}=$ $\inf \left\{\tau: x \pi \tau \in S_{i}, \tau>\frac{1}{2} T\right\}$. Let $C_{i}$ be the simple closed curve composed of $x \pi_{i}\left[0, t_{i}\right]$ and the subarc of $S_{i}$ connecting $x$ and $x \pi_{i} t_{i}$. Clearly this can eventually be done. Finally set $V_{i}=$ int $C_{i}$. Then $\bar{V}_{i} \subset W$ since $W$ is simply connected. $V_{i}$ is either positively invariant or negatively invariant [2, VII, 4.8]. Hence $L_{i}^{+}\left(V_{i}\right) \subset \bar{V}_{i} \subset W$ or $L_{i}^{-}\left(V_{i}\right) \subset \bar{V}_{i} \subset W$. This completes the proof.

ProOF OF THEOREM 4. Let $x$ be a stable isolated critical point and $W$ be a compact simply connected neighborhood of $x$. By Lemmas 5, 6 and 7 there is a net $y_{i}$ in $W$ such that eventually either $L_{i}^{+}\left(y_{i}\right) \subset W$ or $L_{i}^{-}\left(y_{i}\right) \subset W$. 
By the Poincaré-Bendixson Theorem [2, VIII, 1.14], if $L_{i}^{+}\left(y_{i}\right) \subset W$, then $L_{i}^{+}\left(y_{i}\right)$ is a cycle of $\pi_{i}$ or $L_{i}^{+}\left(y_{i}\right)$ contains critical points of $\pi_{i}$. A similar result holds if $L_{i}^{-}\left(y_{i}\right) \subset W$. If $L_{i}^{+}\left(y_{i}\right)\left(\right.$ or $\left.L_{i}^{-}\left(y_{i}\right)\right)$ is a cycle then int $L_{i}^{+}\left(y_{i}\right) \subset W$ and int $L_{i}^{+}\left(y_{i}\right)$ contains a critical point [2, VII, 4.8]. Thus eventually $W$ contains critical points of $\pi_{i}$. Let $x_{i}$ be a critical point of $\pi_{i}$ and assume $x_{i} \rightarrow x_{0}$. Then, for any $t \in R$,

$$
x_{0} \leftarrow x_{i}=x_{i} \pi_{i} t \rightarrow x_{0} \pi t .
$$

Hence $x_{0}$ is a critical point of $\pi$. Since $x$ is an isolated critical point of $\pi$, the desired result easily follows.

REMARKS. (1) If $x$ is a stable critical point of $\pi$ and $x_{i}$ are critical points of $\pi_{i}$ such that $x_{i} \rightarrow x$, it may be that none of the $x_{i}$ are stable. Let $\pi_{n}$ $(n=1,2, \cdots)$ be the planar dynamical system indicated by the following drawing (where the cycle is a circle of radius $1 / n$ ). Then the $\pi_{n}$ can be

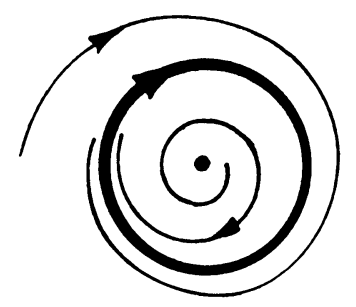

chosen so that they converge to a dynamical system $\pi_{n}$ in which the origin is asymptotically stable.

(2) If $x$ is a critical point of $\pi$, it is possible that there are no critical points of the $\pi_{i}$ close to $x$. Let $\pi_{n}(n=1,2, \cdots)$ be the planar dynamical system given by $\dot{x}=x^{2} /\left(1+x^{2}\right)+1 / n, \dot{y}=0$ and $\pi$ be the planar system given by $\dot{x}=x^{2} /\left(1+x^{2}\right), \dot{y}=0$. Then $\pi_{n} \rightarrow \pi$, each $\pi_{n}$ is free from critical points, and $\pi$ has a critical point at the origin.

\section{REFERENCES}

1. R. C. McCann, Local sections of perturbed local dynamical systems, J. Differential Equations 10 (1972), 336-344.

2. O. Hájek, Dynamical systems in the plane, Academic Press, New York, 1968. MR 39 \#1767.

3. J. Auslander and P. Seibert, Prolongations and stability in dynamical systems, Ann. Inst. Fourier (Grenoble) 14 (1964), fasc. 2, 237-267. MR 31 \#455.

4. N. P. Bhatia and O. Hájek, Theory of dynamical systems. II, Technical Note No. BN-606, University of Maryland, College Park, Md., 1969.

Department of Mathematics and Statistics, Case Western Reserve University, Cleveland, Ohio 44106 